\title{
The Role of Patients' Families in Cancer Treatment Decision-Making \\ Perspectives among Eastern and Western families
}

Abdulrahim Al-Bahri, ${ }^{1}$ Mansour Al-Moundhri, ${ }^{2}$ "Mohammed Al-Azri ${ }^{3}$

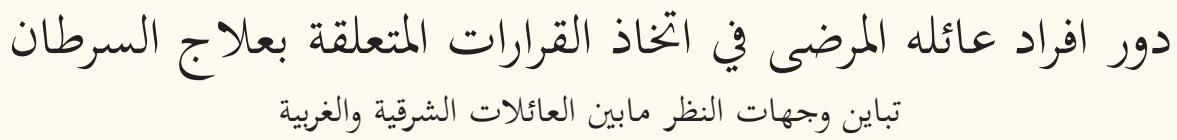

عبد الرحيم البحري، منصور المنذري، محمد العزري

$\mathrm{S}$ HARED TREATMENT DECISION-MAKING (TDM) is the process by which physicians and patients make treatment decisions by sharing information, expressing preferences and agreeing to implement a proposed treatment. ${ }^{1}$ Among patients who are diagnosed with life-threatening diseases such as cancer, shared TDM has been associated with improved physical and psychological health outcomes, greater patient compliance with regards to oncological treatments, higher levels of patient satisfaction and reduced overall healthcare costs. ${ }^{2,3}$ Indeed, it has been observed that the majority of cancer patients prefer having a role in TDM along with their physicians; however, there is controversy regarding the appropriate level of involvement of patients' families. ${ }^{4,5}$

In an online survey of 290 middle-aged urban residents, Alden et al. found that individuals who preferred to be more involved in TDM-either as shared or autonomous TDM-tended to prefer less family involvement for a variety of illnesses ranging in severity, particularly if the family member in question was young. ${ }^{5}$ Interestingly, this study included samples from many Eastern countries, including China, Thailand, South Korea, Malaysia and India; this finding also held in those countries, as well as in the USA and Australia. ${ }^{5}$ Another study found that poor South Korean patients preferred autonomous TDM for end-of-life decisions, as they were often concerned that younger relatives might more heavily weigh financial concerns versus the patient's own preferences. ${ }^{6}$ Researchers in the Asian Pacific region have therefore concluded that there are meaningful segments of patients across very different cultures who prefer little or no family involvement in TDM and higher levels of shared and autonomous TDM. ${ }^{5,6}$ In another study of patient-caregiver dyads in Korea, most patients and caregivers valued and expected family involvement in TDM; however, there was little explicit agreement regarding which party in the dyad should take decisional leadership and which should play a supporting role.?

Several studies have shown that cancer patients and their family members prefer family participation in TDM to some extent. ${ }^{7-9}$ Among patients with advanced lung cancer in Belgium, Pardon et al. reported that patients' family members regularly attended consultation and treatment appointments in order to provide emotional support, request further information and participate in the TDM process. ${ }^{10} \mathrm{~A}$ large, multiregional, prospective cohort study of newly diagnosed patients with lung and colorectal cancer undertaken in the USA found that most participants involved family members in treatment decisions. ${ }^{11}$ Nevertheless, the study concluded that further research is required to evaluate the impact such involvement might have on patient outcomes. The authors also noted that physicians should consider determining the patients' preferences regarding family involvement at the outset of care. ${ }^{11}$ In Oman, many breast cancer patients were found to rely on their families for emotional, physical and financial support, the absence of which negatively affected the patient's prognosis. ${ }^{12}$ It should be noted that the impact of a cancer diagnosis on family members is sometimes similar to that of the patient in terms of stress, depression, anxiety, financial constraints and interpersonal problems. ${ }^{13,14}$

In Eastern countries, patients are more likely to be guided by their families during the TDM process, which is often associated with high levels of positive emotional engagement, and are therefore less likely to make key medical decisions..$^{15}$ Family members in such countries tend to believe that TDM should lie in the hands of the patient's family members and that these individuals should be involved directly in the 
TDM process, perhaps due to a strong sense of moral responsibility. ${ }^{7,16}$ In certain Middle Eastern, North African and South Asian societies, the family has final authority in TDM, as often the patient is inseparably linked to the family as a whole. ${ }^{17}$ For example, the majority of cancer patients in South Korea preferred shared TDM with their family members, although it was noted that there may be disagreements between family members and patients. ${ }^{7}$

In a study from Pakistan, family members sometimes met the physician independently and gave consent for treatment on the patient's behalf. ${ }^{18}$ Furthermore, physicians of cancer patients in China and Japan actually preferred to consult primarily with family members, giving these individuals the authority to decide whether to involve the patient in the TDM process at all. ${ }^{15,19}$ In Oman, some oncologists have noted that family members play a significant role in the TDM process, sometimes to the extent that the patient loses their autonomy; this can delay treatment and even affect survival. ${ }^{20}$ Another study found that, in some Arabic countries, male family members held the dominant role in the TDM process, particularly if the patient was female. ${ }^{21}$

Nevertheless, while most cancer patients seem to prefer family involvement in the TDM process, many physicians in Western countries have raised concerns regarding the potential loss of patient autonomy when family members become involved in treatment. ${ }^{8}$ In such countries, the individual rights of cancer patients are respected and protected by physicians; therefore, family members retain only limited powers when it comes to intervening in medical care, particularly in cases wherein the patient is deemed competent enough to make their own decisions. ${ }^{14,16}$ However, physicians may sometimes encounter family members who ask them not to involve the patient in the TDM process or even, in some cases, not to inform the patient of their diagnosis at all. ${ }^{22}$

Although many physicians welcome varying levels of family involvement in TDM, most families are naturally cautious of challenging the physician's professional authority and intervening with the patient's treatment. ${ }^{23}$ Some physicians could react negatively to family members' requests if they believe them to be in breach of the patient's dignity and/or autonomy. Indeed, medical law in many Western countries states that, in the majority of circumstances, the patients themselves have the final decision with regards to TDM. ${ }^{17}$ However, the cultural background of the physicians may influence their response to involving family members in the TDM process. One study conducted in the USA showed that the majority of physicians of Japanese heritage would obey a family's request not to involve the cancer patient in TDM while very few North American physicians would consent to this request. ${ }^{24}$

In Western countries, the concept of relational autonomy, which considers the individualistic perception of autonomy within a larger social framework, allows for the inclusion of family members in the TDM process. ${ }^{17}$ Thus, physicians are requested to abide by their patients' expressed wishes with regards to family involvement in TDM and to consider the family's opinions during care, in the absence of any evidence of abuse or neglect. ${ }^{23}$ Nevertheless, research conducted in the UK indicated that while the relatives of cancer patients influenced the decision-making process itself, the final decision was ultimately left to the patient. ${ }^{25}$ Similarly, most Australian physicians reported positive attitudes towards family involvement in TDM, but had concerns regarding a number of challenges which can arise when family members are involved in TDM, although the overall benefits of familial involvement were found to outweigh the costs. ${ }^{26}$ Therefore, while family involvement in decision-making is often appreciated, preferences for the nature and extent of their involvement may vary based on other influencing factors. As such, it may be beneficial if physicians ascertain individual patients' preferences and remain open-minded regarding the various ways that the patient's family can be involved.

In summary, TDM in cancer cases is complex, as it involves the patient, the patient's family members and physicians, with each stakeholder potentially having a different opinion regarding the patient's medical needs. When it comes to the degree of acceptance of familial involvement, it seems that the most important factor is not the specific location but the cultural background of those involved. While individuals from Western cultures perceive that the patient's dignity and autonomy must be protected above all, family members of Eastern cultures may not necessarily agree. Further understanding of the differences between Western and Eastern cultures is needed. Moreover, the concept of relational autonomy does not take into consideration the cultural background of the patient or allow family members any legal authority. As such, new legislation may need to be proposed, should family members wish to become more involved in the TDM process in certain countries. However, it is vital that such involvement be considered only in agreement with the patient's wishes as evidenced by a recorded conversation with the provider present, or a legal document (i.e. a power of attorney or advanced legal directive) that specifies the conditions under which the patient desires their family be involved in the TDM process. 


\section{References}

1. Charles C, Gafni A, Whelan T. Decision-making in the physician-patient encounter: Revisiting the shared treatment decision-making model. Soc Sci Med 1999; 49:651-61. doi: 10.1016/S0277-9536(99)00145-8.

2. Whelan T, Levine M, Willan A, Gafni A, Sanders K, Mirsky D, et al. Effect of a decision aid on knowledge and treatment decision making for breast cancer surgery: A randomized trial. JAMA 2004; 292:435-41. doi: 10.1001/jama.292.4.435.

3. Joosten EA, DeFuentes-Merillas L, de Weert GH, Sensky T, van der Staak CP, de Jong CA. Systematic review of the effects of shared decision-making on patient satisfaction, treatment adherence and health status. Psychother Psychosom 2008; 77:219-26. doi: 10.1159/000126073

4. Singh JA, Sloan JA, Atherton PJ, Smith T, Hack TF, Huschka MM, et al. Preferred roles in treatment decision making among patients with cancer: A pooled analysis of studies using the Control Preferences Scale. Am J Manag Care 2010; 16:688-96.

5. Alden DL, Friend J, Lee PY, Lee YK, Trevena L, Ng CI, et al. Who decides: Me or we? Family involvement in medical decision making in Eastern and Western countries. Med Decis Making 2017; 1:272989X17715628. doi: 10.1177/0272989X177 15628.

6. Kim SH. Preferences for autonomy in end-of-life decision making in modern Korean society. Nurs Ethics 2015; 22:228-36. doi: $10.1177 / 0969733014523168$

7. Shin DW, Cho J, Roter DL, Kim SY, Sohn SK, Yoon MS, et al. Preferences for and experiences of family involvement in cancer treatment decision-making: Patient-caregiver dyads study. Psychooncology 2013; 22:2624-31. doi: 10.1002/pon.3339.

8. Laidsaar-Powell R, Butow P, Bu S, Charles C, Gafni A, Fisher A, et al. Family involvement in cancer treatment decision-making: A qualitative study of patient, family, and clinician attitudes and experiences. Patient Educ Couns 2016; 99:1146-55. doi: 10.1016/j.pec.2016.01.014.

9. Burkhalter JE, Bromberg SR. Family-oncologist communication in cancer patient care. Cancer Invest 2003; 21:915-23. doi: $10.1081 / \mathrm{CNV}-120025094$.

10. Pardon K, Deschepper R, Stichele RV, Bernheim JL, Mortier F, Bossuyt $\mathrm{N}$, et al. Preferences of patients with advanced lung cancer regarding the involvement of family and others in medical decision-making. J Palliat Med 2010; 13:1199-203. doi: 10.1089/jpm.2010.0100.

11. Hobbs GS, Landrum MB, Arora NK, Ganz PA, van Ryn M, Weeks JC, et al. The role of families in decisions regarding cancer treatments. Cancer 2015; 121:1079-87. doi: 10.1002/ cncr.29064.

12. Al-Azri MH, Al-Awisi H, Al-Rasbi S, Al-Moundhri M. Coping with a diagnosis of breast cancer among Omani women. J Health Psychol 2014; 19:836-46. doi: 10.1177/1359105313479813.
13. Stenberg U, Cvancarova M, Ekstedt M, Olsson M, Ruland C. Family caregivers of cancer patients: Perceived burden and symptoms during the early phases of cancer treatment. Soc Work Health Care 2014; 53:289-309. doi: 10.1080/0098 1389.2013.873518.

14. Cherry MJ. Re-thinking the role of the family in medical decision-making. J Med Philos 2015; 40:451-72. doi: 10.1093/ jmp/jhv011.

15. Kitayama S, Park H, Sevincer AT, Karasawa M, Uskul AK. A cultural task analysis of implicit independence: Comparing North America, Western Europe, and East Asia. J Pers Soc Psychol 2009; 97:236-55. doi: 10.1037/a0015999.

16. Bian L. Medical individualism or medical familism? A critical analysis of China's new guidelines for informed consent: The basic norms of the documentation of the medical record. J Med Philos 2015; 40:371-86. doi: 10.1093/jmp/jhv016.

17. Gilbar R, Miola J. One size fits all? On patient autonomy, medical decision-making, and the impact of culture. Med Law Rev 2015; 23:375-99. doi: 10.1093/medlaw/fwu032.

18. Humayun A, Fatima N, Naqqash S, Hussain S, Rasheed A, Imtiaz $\mathrm{H}$, et al. Patients' perception and actual practice of informed consent, privacy and confidentiality in general medical outpatient departments of two tertiary care hospitals of Lahore. BMC Med Ethics 2008; 9:14. doi: 10.1186/14726939-9-14.

19. Sekimoto M, Asai A, Ohnishi M, Nishigaki E, Fukui T, Shimbo T, et al. Patients' preferences for involvement in treatment decision making in Japan. BMC Fam Pract 2004; 5:1. doi: 10.1186/ 1471-2296-5-1.

20. Burney IA. The trend to seek a second opinion abroad amongst cancer patients in Oman: Challenges and opportunities. Sultan Qaboos Univ Med J 2009; 9:260-3.

21. Moghadam VM. Patriarchy in transition: Women and the changing family in the Middle East. J Comp Fam Stud 2004; $35: 137-62$.

22. McCabe MS, Wood WA, Goldberg RM. When the family requests withholding the diagnosis: Who owns the truth? J Oncol Pract 2010; 6:94-6. doi: 10.1200/JOP.091086.

23. Ho A. Relational autonomy or undue pressure? Family's role in medical decision-making. Scand J Caring Sci 2008; 22:128-35. doi: 10.1111/j.1471-6712.2007.00561.x.

24. Gabbay BB, Matsumura S, Etzioni S, Asch SM, Rosenfeld KE, Shiojiri T, et al. Negotiating end-of-life decision making: A comparison of Japanese and U.S. residents' approaches. Acad Med 2005; 80:617-21.

25. Gilbar R. Family involvement, independence, and patient autonomy in practice. Med Law Rev 2011; 19:192-234. doi: 10.1093/medlaw/fwr008

26. Laidsaar-Powell R, Butow P, Bu S, Fisher A, Juraskova I. Oncologists' and oncology nurses' attitudes and practices towards family involvement in cancer consultations. Eur J Cancer Care (Engl) 2017; 26:e12470. doi: 10.1111/ecc.12470. 